\title{
Integrating motion sensing and wearable, modular high-density diffuse optical tomography: preliminary results
}

Brigadoi, Sabrina, Ganglani, Aman, Zhao, Hubin, Cooper, Robert

Sabrina Brigadoi, Aman Ganglani, Hubin Zhao, Robert J. Cooper, "Integrating motion sensing and wearable, modular high-density diffuse optical tomography: preliminary results," Proc. SPIE 11074, Diffuse Optical Spectroscopy and Imaging VII, 1107405 (11 July 2019); doi: 10.1117/12.2527197

SPIE. Event: European Conferences on Biomedical Optics, 2019, Munich, Germany 


\title{
Integrating motion sensing and wearable, modular high-density diffuse optical tomography: Preliminary results
}

\author{
Sabrina Brigadoi*a ${ }^{* a}$ Aman Ganglani ${ }^{\mathrm{b}}$, Hubin Zhao ${ }^{\mathrm{b}, \mathrm{c}}$, Robert J. Cooper ${ }^{\mathrm{b}, \mathrm{c}}$ \\ ${ }^{a}$ Department of Developmental Psychology, University of Padova, Padova, Italy; ${ }^{b}$ Department of \\ Medical Physics and Biomedical Engineering, UCL, London, UK; ${ }^{c}$ neoLAB, The Evelyn Perinatal \\ Imaging Centre, Rosie Hospital, Cambridge, UK \\ *sabrina.brigadoi@unipd.it; phone 00390498276538
}

\begin{abstract}
The next generation of diffuse optical imaging systems will consist of wearable and fiber-less devices, to exploit the advantages of diffuse optical imaging over other functional neuroimaging techniques and meet the needs of users to acquire data in real-world settings. Recently, research at UCL gave rise to a novel, modular high-density diffuse optical tomography (DOT) system that was validated by reconstructing activation images over the motor cortex of a thumb-tofinger extension task. The real question, however, is whether these fiber-less systems can be employed whilst the subject performs real-world activities, that is, whether they can provide reliable signals during participant motion. Integrating motion sensors into modular wearable electronics is straightforward. In this study we acquired DOT and motion sensor data whilst participants performed different activities involving motion. In one acquisition, only accelerometer data were acquired while in the second acquisition, all 9-axis of data (accelerometer, gyroscope and magnetometer data) were acquired. Results demonstrated that acceleration data from motion sensors is not enough to detect motion artifacts whilst performing active movement (e.g., walking), since the global motion obscures any subtle motion artifact. Conversely, by combining accelerometer and gyroscope data it seems possible to detect motion artifacts even during walking, that is when a global motion is present. However, not all types of motion artifacts (e.g., eyebrow raising) could be detected even with this full data configuration. Further studies are required to shed light on this important research question.
\end{abstract}

Keywords: DOT, motion sensor, wearable DOT, MPUs

\section{INTRODUCTION}

One of the main advantages of diffuse optical imaging devices is their portability and the possibilities they offer to monitor brain activity in real-world settings and whilst performing real-world activities (e.g., walking, sport, etc.) ${ }^{1,2}$. This flexibility would not be possible with other brain imaging techniques, such as functional magnetic resonance imaging, where participants are constrained to lie motionless inside a scanner. Recently, research at UCL gave rise to a novel, modular, fiber-less high-density diffuse optical tomography (DOT) system. Its performance was validated with a thumbto-finger extension task, which showed a clear activation of the motor cortex in each participant ${ }^{3}$. Fiber-less systems should reduce the occurrence of motion artifacts in the recorded signals, avoiding any artifact caused by decoupling between scalp and optodes due to fiber movements. However, an important question is whether these fiber-less systems can be truly employed to image the brain whilst the subject performs real-world activities, that is whether they can provide reliable signals during motion. One additional advantage of modular wearable electronics is the opportunity to integrate motion sensors into the modules, to provide an auxiliary signal that could be used in DOT data processing to regress potential motion artifacts. The aim of the present study was to investigate the relationship between motion artifacts identified in DOT data and the signal provided by integrated motion sensors, to understand the benefits and impact that the integration of the two could provide.

\section{MATERIAL AND METHODS}

\subsection{Hardware set-up and experimental paradigm}

Two acquisitions were devised to investigate this issue. In experiment A, eleven participants were monitored with a fiber-less high density DOT system consisting of 12 DOT modules, located bilaterally over the somatomotor cortex.

Diffuse Optical Spectroscopy and Imaging VII, edited by Hamid Dehghani, Heidrun Wabnitz, Proc. of SPIE-OSA Vol. 11074, 1107405 · @ 2019 SPIE-OSA · CCC code: 1605-7422/19/\$21 · doi: 10.1117/12.2527197 
Each module was composed of 4 detectors and 2 sources and a 9-axis motion sensor (but only the norm of the 3 acceleration axes was recorded). Participants underwent two imaging sessions, one while seated and the other while walking. The aim of this experiment was to investigate whether the norm of the 3 axis acceleration is enough to detect motion artifacts in the DOT signals during participant's motion. In experiment B, one participant was monitored with a fiber-less system consisting of 2 DOT modules. Each module was composed of 4 detectors and 2 sources and an MPU9250 InvenSense 9-axis motion sensor. One module was positioned on the forehead at Fpz (according to the 10-20 EEG system), while the other one was positioned just below Pz. Two additional independent SparkFun Razor 9-axis motion sensors were placed outside the two DOT modules, and glued directly to the skin. These contained the same InvenSense MPU-9250 motion sensor chip which was programmed to have identical operating parameters as the DOT modules. A USB cable was used to power and transmit data from these additional MPUs. The participant was asked to perform a series of movements at different speeds whilst seated or walking (moving the head left/right/up/down, eyebrows raising, speaking). The aim of this second experiment was to investigate the information provided by 9 -axis motion sensors relative to motion artifacts present in the DOT data.

\subsection{Data analysis}

Matlab was used to simultaneously control the experimental paradigm and data acquisition from the two external MPUs by utilizing the parallel computing toolbox. Data from the DOT modules were acquired using Visual Studio. Raw motion sensor data were converted into units of $g$ for the accelerometer data (axes 1 to 3), degrees per second for the gyroscope data (axes 4 to 6) and Gauss for the magnetometer data (axes 7 to 9). In Experiment A, only accelerometer data were available.

A)
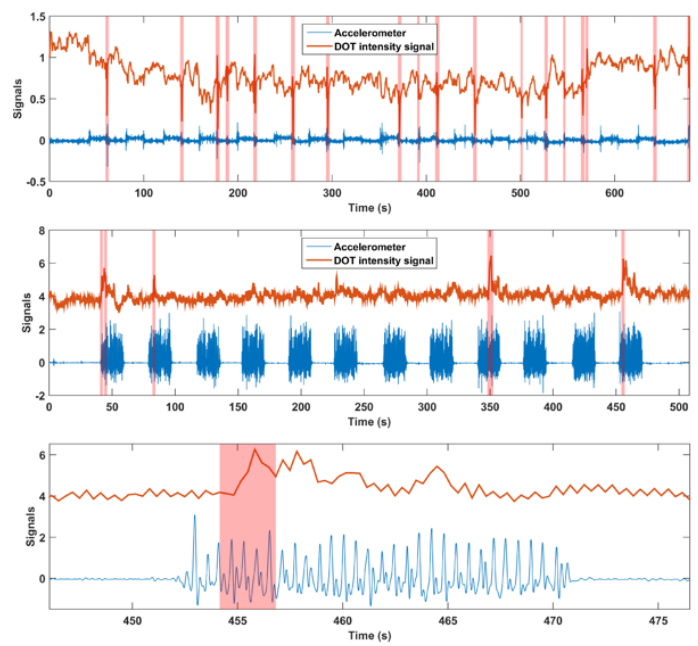

B)
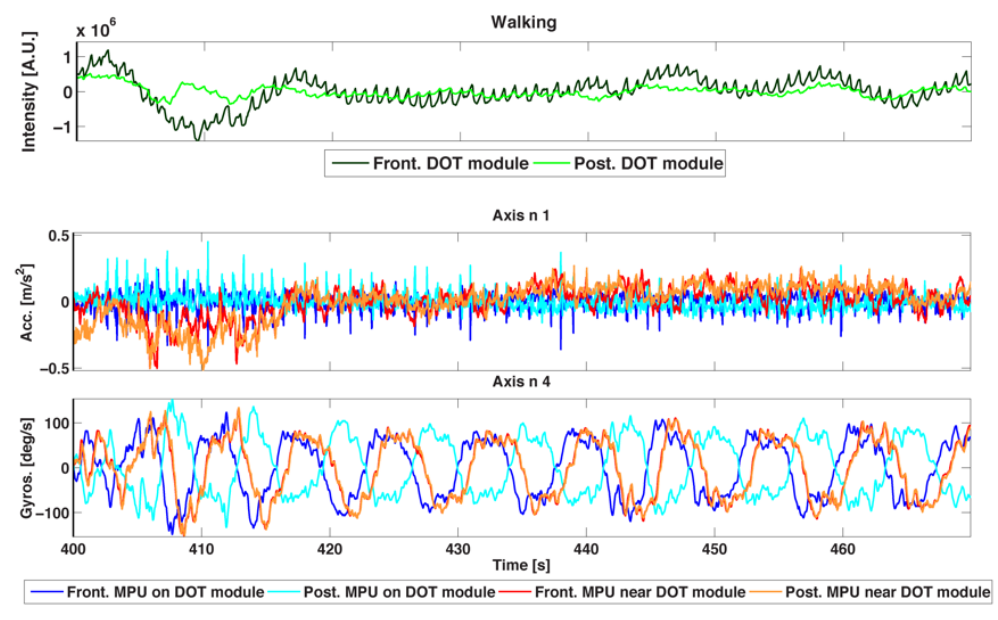

Figure 1. A) Example of DOT and accelerometer signal for a seated participant (upper panel) and a walking participant (middle panel) of Experiment A. The bottom panel is a zoomed in version of the last motion artifact of the middle panel. Shaded areas represent the identified motion artifacts. B) Example of DOT signals (upper panel) and motion sensor signals (one axis of accelerometer and gyroscope data for all 4 MPUs) of Experiment B. The participant was walking without performing any other task. The data from all four MPUs and two exemplary DOT channels from both DOT modules (frontal and posterior) are reported with different colors.

\section{RESULTS}

We found a good correspondence between the presence of small DOT motion artifacts and accelerometer spikes when the participant was seated in experiment A, when only the norm of the accelerometer data was available. This was however not the case during the walking task, since the movement of the sensor was dominated by the global walking movement (Fig. 1A). These results demonstrated that acceleration data from motion sensors alone are not enough to detect motion artifacts whilst performing active movement (e.g., walking), since the global motion will obscure any subtle motion artifact. Results of experiment B revealed that the 9-axis motion sensors, both on and near the DOT module, provided more useful and interesting information, as also recently shown by Siddiquee et $\mathrm{al}^{4}$, being able to detect most of the elicited motion artifacts even during walking (Fig. 1B, 2). By combining accelerometer and gyroscope 
data it seems possible to detect motion artifacts even during walking, that is when a global motion is present. However, not all types of motion artifacts could be detected: eyebrow raising was still undetected in the motion sensor data, while motion artifacts could be clearly seen in the DOT recording (Fig. 2A). Additional motion sensors placed near the DOT module seemed to provide sometimes complementary information to the integrated motion sensors. As expected, almost no motion artifact could be seen in the recorded DOT module while walking alone (Fig. 1), highlighting the good quality and utility of this wearable device.
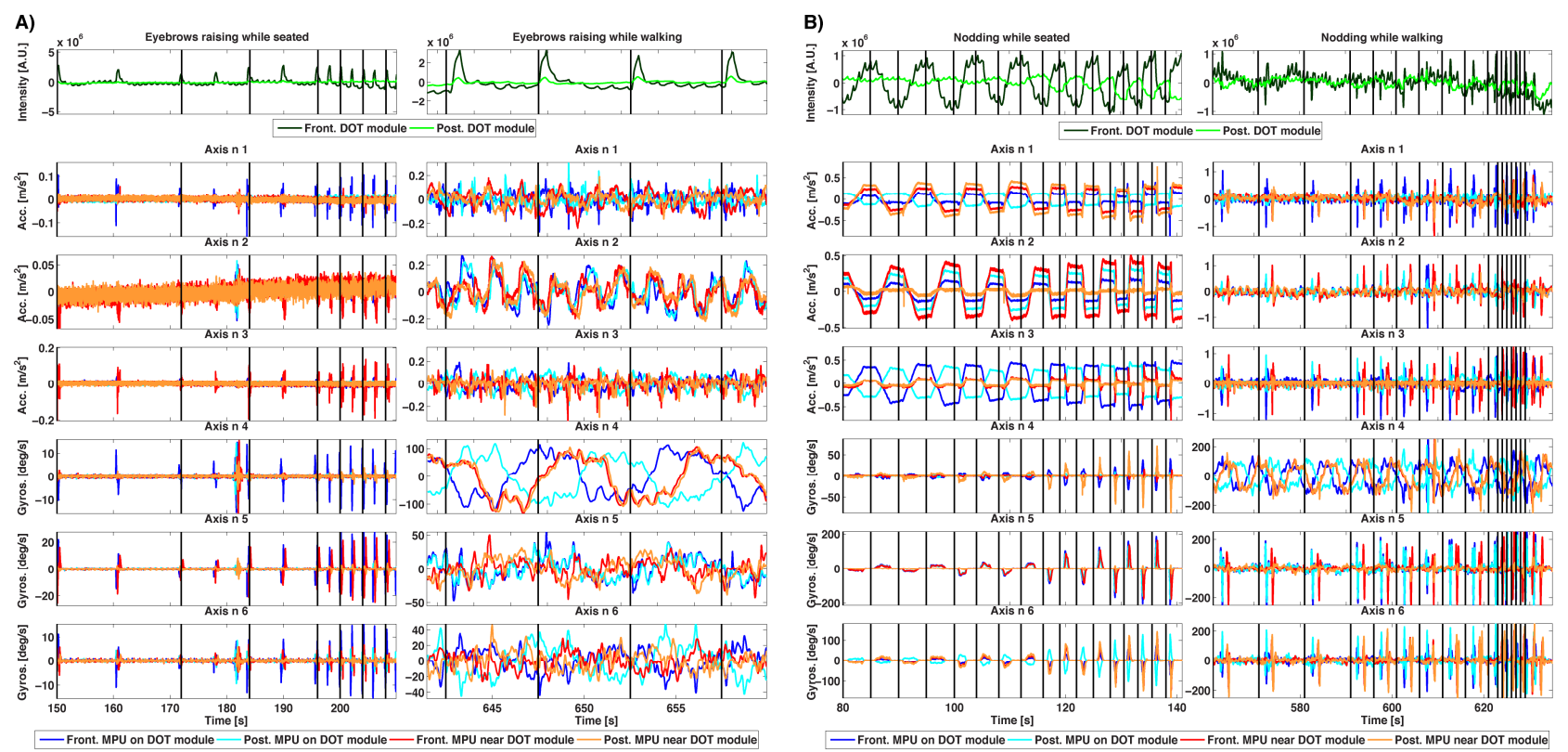

Figure 2. Example of DOT signals (upper panel) and motion sensor signals (accelerometer and gyroscope data on all 3 axes) of Experiment B during A) the raising eyebrows task and B) the nodding task. The left column of each panel reports the data acquired whilst the participant was seated, whereas the right column the data acquired whilst the participant was walking. The data from all four MPUs and two exemplary DOT channels from both DOT modules (frontal and posterior) are reported with different colors.

\section{CONCLUSIONS}

Our preliminary results revealed that 9-axis motion sensors seem necessary when planning to perform experiments involving walking. Further studies are required to investigate how to integrate these motion sensor data inside the DOT reconstruction pipeline.

\section{REFERENCES}

[1] Cutini, S. and Brigadoi, S., "Unleashing the future potential of functional near-infrared spectroscopy in brain sciences," J. Neurosci. Methods 232, 152-156 (2014).

[2] Zhao, H. and Cooper, R. J., "Review of recent progress toward a fiberless, whole-scalp diffuse optical tomography system," Neurophotonics 5(01), 1 (2017).

[3] Chitnis, D., Cooper, R. J., Dempsey, L., Powell, S., Quaggia, S., Highton, D., Elwell, C., Hebden, J. C. and Everdell, N. L., "Functional imaging of the human brain using a modular, fibre-less, high-density diffuse optical tomography system,” Biomed. Opt. Express 7(10), 4275 (2016).

[4] Siddiquee, M. R., Marquez, J. S., Atri, R., Ramon, R., Perry Mayrand, R. and Bai, O., "Movement artefact removal from NIRS signal using multi-channel IMU data,” Biomed. Eng. Online 17(1), 120 (2018). 